\title{
Retroperitoneal Appendicitis: A Surgical Dilemma
}

\section{Basil George Verghese, Sanjana Kalvehalli Kashinath, R Ravikanth}

\section{ABSTRACT}

Introduction: Acute appendicitis is frequently encountered in both the surgical OPD and emergency room setting. But, a retroperitoneal appendicitis is very rarely seen and the literature available on it is scarce.

Case report We wish to presenta case of a 26 years old female who presented to the emergency room with features of appendicitis and on whom an appendectomy was done. But during the surgery, the appendix was found situated retroperitoneally and had to be removed via a retrograde approach. We followed it with a short discussion of retroperitoneal appendix, the presentation of retroperitoneal appendicitis and its surgical management.

Conclusion: Documented cases of retroperitoneal appendix are rare to the best of our knowledge and should be kept as a differential when a patient presents with atypical features of appendicitis.

Keywords: R etroperitoneal appendix, R etrograde appendectomy, Abnormal positions of the appendix.

How to cite this article: Verghese BG, Kashinath SK, Ravikanth R. R etroperitoneal Appendicitis: A Surgical Dilemma. Euroasian J Hepato-G astroenterol 2013;3(1):83-84.

\section{Source of support $\mathrm{Nil}$}

\section{Conflict of interest: None}

\section{CASE REPORT}

A 26-year-old female, married, having one child presented to our hospital emergency room with an $\mathrm{h} / \mathrm{o}$ low backache since 3 days, pain in the right lower abdomen since 2 days and a single episode of vomiting the previous day. She revealed that she had similar episodes in the past which subsided after taking over the counter analgesics.

On examination her temperature was $98.6^{\circ} \mathrm{F}$, pulse was $86 / \mathrm{min}$, blood pressure was $130 / 70 \mathrm{~mm} \mathrm{Hg}$. Her last menstrual period was 14 days back, 5/28, regular with normal flow. Per abdomen examination elicited tenderness in the right iliac fossa (RIF). Psoas sign was positive. Rest of the systemic examination was unremarkable.

L aboratory values revealed an $\mathrm{Hb}$ of $11.4 \mathrm{gm} \%$, a total count of 7,500 cells/cumm and a differential count of $51 \%$ polymorphs and $49 \%$ lymphocytes. U rine microscopy showed 3 to 5 pus cells/hpf. Rest of the laboratory values were within their respective normal parameters. A $n$ ultrasound of the abdomen showed no collection in the RIF but the appendix was not visualized. Due to financial constraints of the patient, we were unable to perform a computed tomographic (CT) scan of the abdomen.

A provisional diagnosis of acute appendicitis was made with urinary tract infection as the main differential. The patient was admitted and managed conservatively on analgesics and intravenous antibiotics for 8 hours.
Despite this there was only a minimal reduction in the patient's symptoms. A urine sample was sent for culture and sensitivity studies which subsequently reported no growth. The patient was posted for an open appendectomy for the following day.

During surgery, minimal collection of fluid was noted in the RIF, but the appendix was found missing (Fig. 1). Consequently, the cecum was mobilized by opening the lateral peritoneal reflection (Fig. 2) which revealed the appendix situated retroperitoneally (Fig. 3).

There was edema present of the surrounding retroperitoneal structures. The appendix was around $4 \mathrm{~cm}$ in length, inflamed but intact with no signs of rupture. The base of the appendix was healthy. A s the tip of the appendix was not visual ized, a retrograde appendectomy was performed and the appendix specimen was sent for a histopathological study. The biopsy reported a congestion and presence of acute inflammatory cells in the walls of the appendix consistent with an impression of acute appendicitis. No abdominal drain was placed, patient was nil by mouth for the next 24 hours.

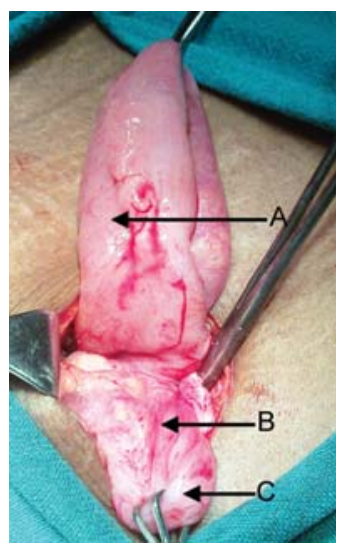

Fig. 1: Appendix not found at the ileocecal junction, (A) distal ileum, (B) confluence of tenia, (C) cecum

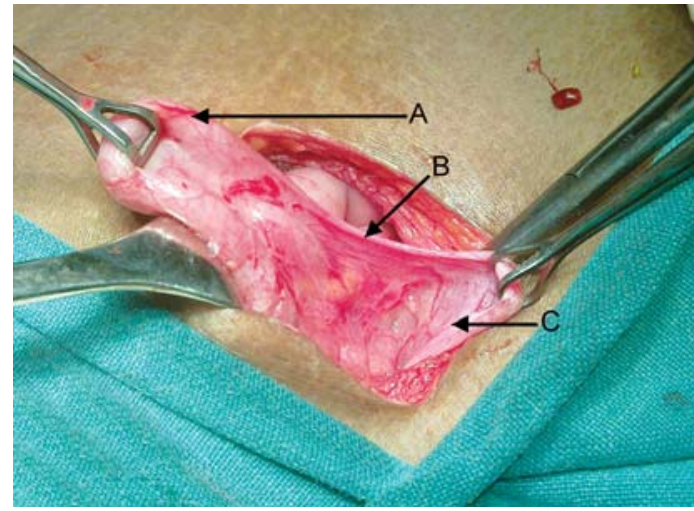

Fig. 2: R eflection of the peritoneum after mobilizing the cecum, (A) cecum, (B) peritoneal reflection, (C) distal ileum 


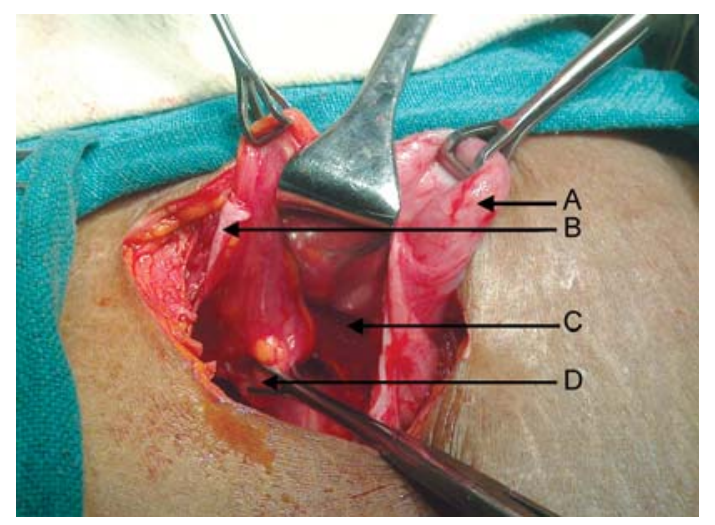

Fig. 3: Appendix located retroperitoneally, (A) distal ileum, (B) peritoneal reflection, $(C)$ retroperitoneum, $(D)$ base of appendix

The postoperative recovery of the patient was uneventful. She was discharged after 3 days and review ed in the surgical OPD 3 days later for suture removal.

\section{DISCUSSION}

It was Reginald Fritz who first coined the term appendicitis in 1886 and recommended early surgical treatment for the disease. $^{1}$

The appendix is located at the convergence of the tenia along the inferior aspect of the cecum. The tip of the appendix may lie in a variety of positions. The most common location is retrocecal $77 \%$. It is pelvic in $30 \%$ and retroperitoneal in $7 \%$ of the population. ${ }^{2}$ These anatomic variations might be responsible for atypical presentations of appendicitis. ${ }^{3}$ There may be a congenital absence of the appendix which is very rare and seen approximately in $1 / 1,00,000$ laparotomies. ${ }^{4}$

A retroperitoneal appendix is relatively liable to disease because it is cut off from direct superior mesenteric circulation. ${ }^{5}$ The onset of pain usually begins in the epigastrium, nausea and vomiting is frequently noted, but pain at M cBurney's point is modified or absent. ${ }^{6}$

On physical examination the classical sign for a retroperitoneal appendix is the iliopsoas sign where the patient has pain on the extension of the right hip. Other signs which are useful but seldom elicited include:

1. Dunphy's sign: Pain on coughing.

2. Rovsing's sign: Pain in RIF on pal pation on the left iliac fossa.

3. Obturator sign: Pain in the hypogastrium on internal rotation and flexion of hip suggestive of a pelvic appendix.

4. Pointing sign: Here, the patient is asked to show where the pain first migrated and where it subsequently migrated.

The white cell count is elevated with a predominantly neutrophilic picture. A completely normal count and differential is seen in $10 \%$ of the cases. ${ }^{7} \mathrm{M}$ inimal pyuria maybe present as the infected appendix may come in contact with the ureter and cause its inflammation. ${ }^{8}$
UItrasound with a sensitivity of $85 \%$ and a specificity of $>90 \%$ is the preferred imaging modal ity in children ${ }^{9}$ while a CT scan is best in adults and in the elderly. ${ }^{10}$

It is hazardous to remove the appendix through the normal incision used in appendicectomy because there is high probability of rupturing the appendix. ${ }^{5} \mathrm{~A}$ laparoscopic appendicectomy is not possible either as the tip of the appendix is not visualized. In these cases, a retrograde appendectomy is the surgery of choice where the appendix is approached and isolated via a lateral peritoneal incision after mobilizing the right colon.

\section{CONCLUSION}

The presence of a retroperitoneal appendix should be on a surgeons mind when dealing with a patient with abdominal pain with atypical features of appendicitis and a retrograde appendectomy is the surgery of choice for a retroperitoneal appendix.

\section{REFERENCES}

1. Nesbit RR Jr, Fitz RH. (1843-1913): A bio-bibliography [BA thesis]. Cambridge, M A : Harvard College 1961;165.

2. Prystowsky JB, Pugh CM, Nagle AP. Current problems in surgery: A ppendicitis. Curr Prob Sug 2005;42:688-742.

3. Poole GV . A natomic basis for delayed diagnosis of appendix. South M ed J 1990;83:771-73.

4. Zetina-M ejía CA, A Ivarez-CosíoJE, Quillo-Olvera J Congenital absence of the cecal appendix. Case report. Cir Cir 2009 SepOct;77(5):407-10.

5. Small AB. The retroperitoneal appendix. Texas State J M 192829;24:550-55.

6. M arbury WB. The retroperitoneal (retrocolic) appendix. Ann Sug 1938 M ay;107(5):819-28.

7. Fitz RH. Perforating inflammation of the vermiform appendix with special reference to its early diagnosis and treatment. Am J Med Sci 1886; 92:321-46.

8. Donald DC. A cute retroperitoneal appendicitis. South Med J 1925;18(5):364-67.

9. B renner $D, E$ Elliston $C, H$ all $E$, et al. Estimated risks of radiation induced fatal cancer from pediatric CT. A m J Roentgenol 2001;176:289-96.

10. W eltman DI, Y u J, K rumenacker J J r, et al. Diagnosis of acute appendicitis. Comparison of 5 to $10 \mathrm{~mm} \mathrm{CT}$ sections in the same patient. Radiology 2000;216:172-77.

\section{ABOUT THE AUTHORS}

\section{Basil George Verghese (Corresponding Author)}

Junior Resident, Department of M edicine, St. Johns Hospital K attappana, Idukki, K erala, India, e-mail: drbasilgeorge@ gmail.com

\section{Sanjana Kalvehalli Kashinath}

J unior Resident, Department of Pediatrics, CG Hospital, Davangere Karnataka, India

\section{R Ravikanth}

Consultant Surgeon, Department of Surgery, St. Johns Hospital K attappana, Idukki, K erala, India 